\title{
Development of cost-effective loT module-based pipe classification system for flexible manufacturing system of painting process of high- pressure pipe
}

\author{
Young Jun Yoo ( $\nabla$ youdalj@kitech.re.kr) \\ Korea Institute of Industrial Technology https://orcid.org/0000-0003-4898-7513
}

\section{Research Article}

Keywords: Painting process, Pipe classification, Internet of Things, Flexible manufacturing system

Posted Date: August 3rd, 2021

DOl: https://doi.org/10.21203/rs.3.rs-723541/v1

License: (9) This work is licensed under a Creative Commons Attribution 4.0 International License. Read Full License

Version of Record: A version of this preprint was published at The International Journal of Advanced Manufacturing Technology on January 5th, 2022. See the published version at https://doi.org/10.1007/s00170-021-08478-1. 


\title{
Development of cost-effective IoT module-based pipe \\ classification system for flexible manufacturing system of painting process of high-pressure pipe
}

\author{
Young-Jun, Yoo
}

Received: date / Accepted: date

\begin{abstract}
This paper researches an IoT module-based pipe classification system for a flexible manufacturing system that recognizes the size and length of pipes used in the painting process of high-pressure pipes. The proposed system is composed of an IoT module, USB camera, and edge TPU for pipe classification. The proposed system recognizes the type of pipe by three processes; object detection of the pipe, line detection algorithm of the three regions of interest, and pipe classification algorithm based on the line detection algorithm. Furthermore, the proposed system enables web-based real-time monitoring, providing convenience to workers and helping them make quick decisions. The IoT module interfaces with the painting robot and the sequence control that paints for each type of pipe is executed in the painting robot,
\end{abstract}

Young-Jun, Yoo

Korea Inst. Industrial Tech. (KITECH), 89, Yangdaegiro-gil, Ipjang-myeon, Seobuk-gu, Cheonan-si, Chungcheongnam-do, Republic of Korea.

youdalj@kitech.re.kr 
allowing flexible manufacturing of the painting process.

Keywords Painting process - Pipe classification $\cdot$ Internet of Things $\cdot$ Flexible manufacturing system

\section{Introduction}

Drilling refers to the operation of drilling a hole up to several tens of kilometers underground to produce oil and gas underground, and in this case, pipes and valves capable of withstanding high-pressure (15,000 psi) are required. High-pressure pipes have threaded, flanged, or union connectors at both ends and are widely used in oil drilling and production, splicing, and breaking and testing operations. Additionally, a high-pressure pipe with the straight tube with union connections at both ends is widely used to deliver high-pressure flow in break manifolds, junction manifolds, maintenance manifolds, and test manifolds.

A flexible manufacturing system is a system that automates production to manufacture various products with high productivity flexibly. In order to cope with the mix of different part types and the production volume adjusted according to changes in demand patterns, it is a productivity that simultaneously aims for flexibility and productivity of production that can process various parts types simultaneously. The goals pursued by the flexible production system are essentially flexibility, productivity, and reliability. In recent years, mass production and traditional manufacturing industries such as chemical and machinery, represented by fixed equipment, are also introduced for survival. Recently, the flexible production system is spreading as the 
industrial Internet, multi-functional robots, and big data technologies improve part identification ability, change processes quickly and significantly reduce setup costs.

Recently, numerous studies $[1--3]$ have been conducted on flexible production. . This paper [1] presents a general architecture of digital-twin visualization for flexible manufacturing systems (FMS) and for developing virtual digital-twin scenes architecture. Furthermore, the design of non-centralized control architectures is proposed [2] to improve their energy efficiency and promote flexibility. Also, seamless HumanComputer-Machine Interaction architecture is presented [3] to support the operator's supervision activity with flexible manufacturing systems.

In expanding various identification ability improvement systems for flexible production, the possibility of recognition systems using deep learning is expanding recently. In computer vision, deep learning is most actively applied and developed, and it is actively used in recognition systems for flexible production. Among them, object detection is to detect a specific element in an image. Object recognition models such as VGG-SSD [4], MobileNet-SSD [5], Faster-RCNN [6], and YOLO [7] can be used in object detection. Object detection detects an object and its bounding box in an image. This deep learning model receives an image as input and outputs the bounding box, class list, and class confidence. Object detection is used in various industrial fields for autonomous driving, detecting abnormal situations in the medical field, and detecting defective parts in the manufacturing industry [8-11].

This paper intends to improve the quality of the painting process, which is the final finishing process, among the manufacturing process for the production of highpressure pipe joints that can connect pipes without welding, which is used for drilling. 
Existing 2-inches and 3-inches products are pre-produced and sold products, and workers are performing undercoating and $4 \sim 6$ times of intermediate and top coating simultaneously. In this series of manufacturing processes, the painting and packaging process is currently being carried out manually. In particular, in the case of painting, the worker sprays the pipe, which is the work object, with a painting gun while considering the quality of the film thickness and is performed by simple repetitive work.

The IoT module developed in this paper is applied to a flexible manufacturing system for the painting process of the high-pressure pipe to improve the poor working environment of the conventional manual process. The proposed painting process makes the coating quality uniform, dramatically improves productivity, and shortens the drying time. The proposed system recognizes nine types of pipes, three types of diameter ( 2 inches, 3 inches, and 4 inches) and three types of lengths (6ft., $8 \mathrm{ft}$., and 10ft.), and informs the recognition results to the gripping robot and painting robot. Also, this paper presents the recognition algorithms for the classification of the pipe and a web-based monitoring system for monitoring results. By establishing a system that recognizes pipes regardless of the type of painted pipe, flexible production of the painting process is possible.

This paper is organized as follows. Section II describes the conventional painting process of the high-pressure pipe. Section III presents the proposed system and process. Section IV provides the method validation setup of the proposed methodology. Section V shows the results using the proposed process. In Section VI, the conclusion is presented. 

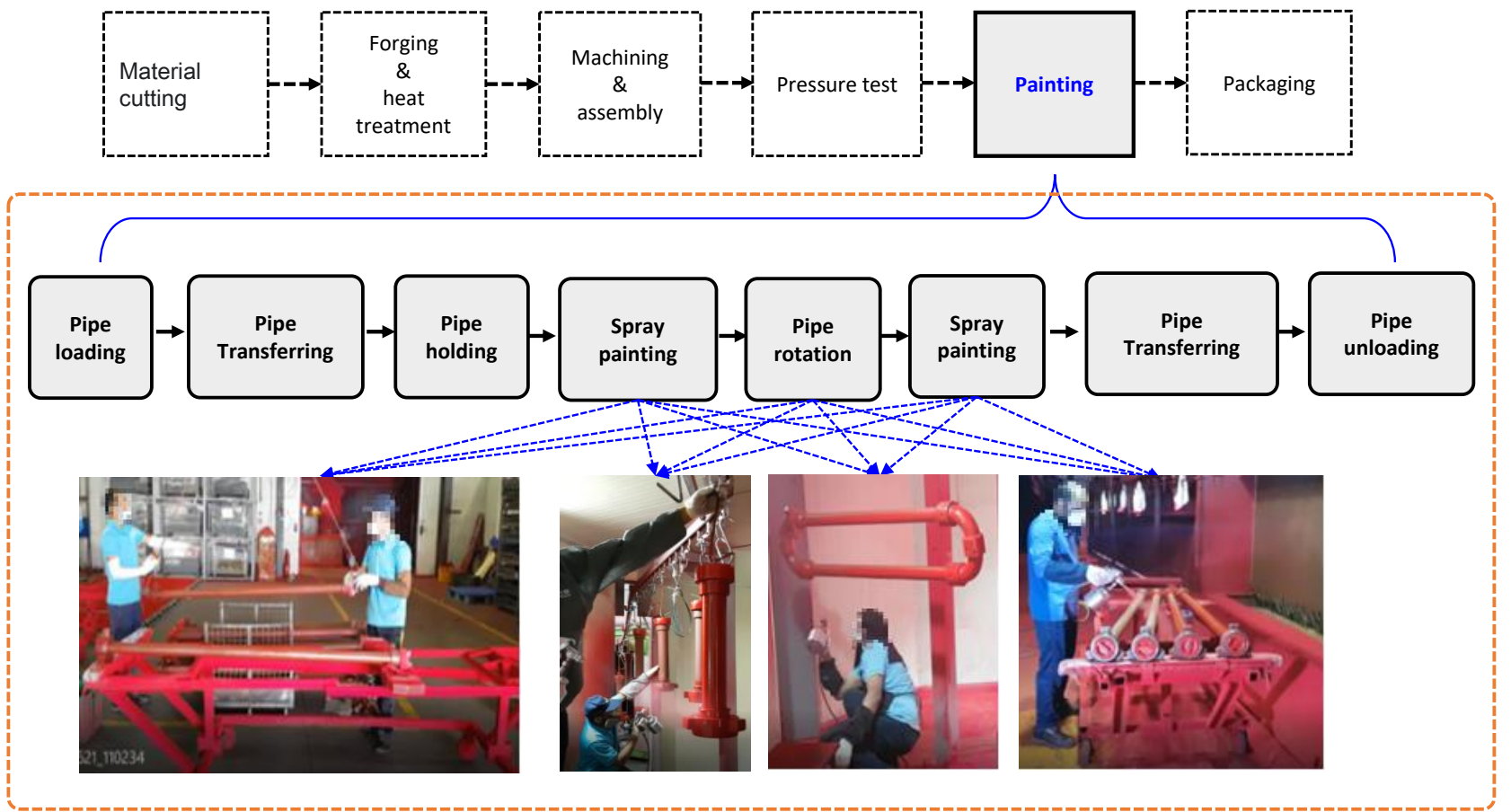
Table 1 Conventional manufacturing process and equipment corresponding to the process.

\begin{tabular}{ll} 
Process & Equipment \\
\hline \hline Cutting & CNC \\
\hline Machining & CNC \\
\hline Assembly & H/Machine \\
\hline Pressure Test & H/T Machine \\
\hline Painting & Manual \\
\hline Packaging & Manual \\
\hline
\end{tabular}

\section{Conventional painting process}

A high-pressure (15,000 psi) pipe manufacturing for oil and gas drilling consists of material cutting, forging, heat treatment, machining, assembling, pressure testing, painting, and packaging, as shown in figure 1 The high-pressure $(15,000 \mathrm{psi})$ pipe manufacturing for oil and gas drilling consists of material cutting, processing, assembling, pressure testing, painting, and packaging. This paper aims to improve the coating process during the manufacturing process of high-pressure pipes. As shown in figure 2, the painting process loads the pipe onto the conveyor belt, transports it, and then fixes the pipe. After manually spray painting the fixed pipe up to $4 \sim 6$ times, the pipe is transported on a conveyor belt for packaging. Additionally, Table 1 shows equipment corresponding to the pipe manufacturing process.

In this series of manufacturing processes, painting and packaging processes are currently being performed manually (The painting process in figure 1. In particular, in the case of painting, the operator sprays the paint onto the pipe, which is the work object, with the painting gun, while the operator repeatedly moves the nozzle gun to 
the left and right while watching the painting thickness.

The operator removes oil and foreign substances after processing and assembly before the middle/top coating work and performs the undercoat coating on the outer surface of the pipe (Pup Joint) using a brush. After that, the worker puts the pipe on the transport cart for intermediate/top coating, moves the product on the transport cart to the painting booth, rotates the pipe by hand about six times, and sprays the middle/top coating with a paint gun. After painting, the truck is moved to unload the product, and the operator completes the touch-up painting work with a brush on the unpainted section while visually checking it. After finishing the painting, the pipe is dried for more than 40 minutes to dry.

In this paper, we propose a flexible productivity improvement system for the painting process of high-pressure pipe using an IoT module. Through the proposed system, it is possible to improve the poor working environment of the manual painting process, make the coating quality uniform, dramatically improve productivity, reduce drying time, and reduce the amount of paint.

For automatically painting the pipe, it is necessary to recognize the length and diameter of the pipe. The existing method (figure 1) uses a proximity sensor to recognize the thickness and length as it passes by. The problem with the existing method is that it is easy to apply when the length and diameter of the pipe are limited. However, when the length and diameter are diversified, there is a problem that a proximity sensor must be installed, and each interface must be applied. 


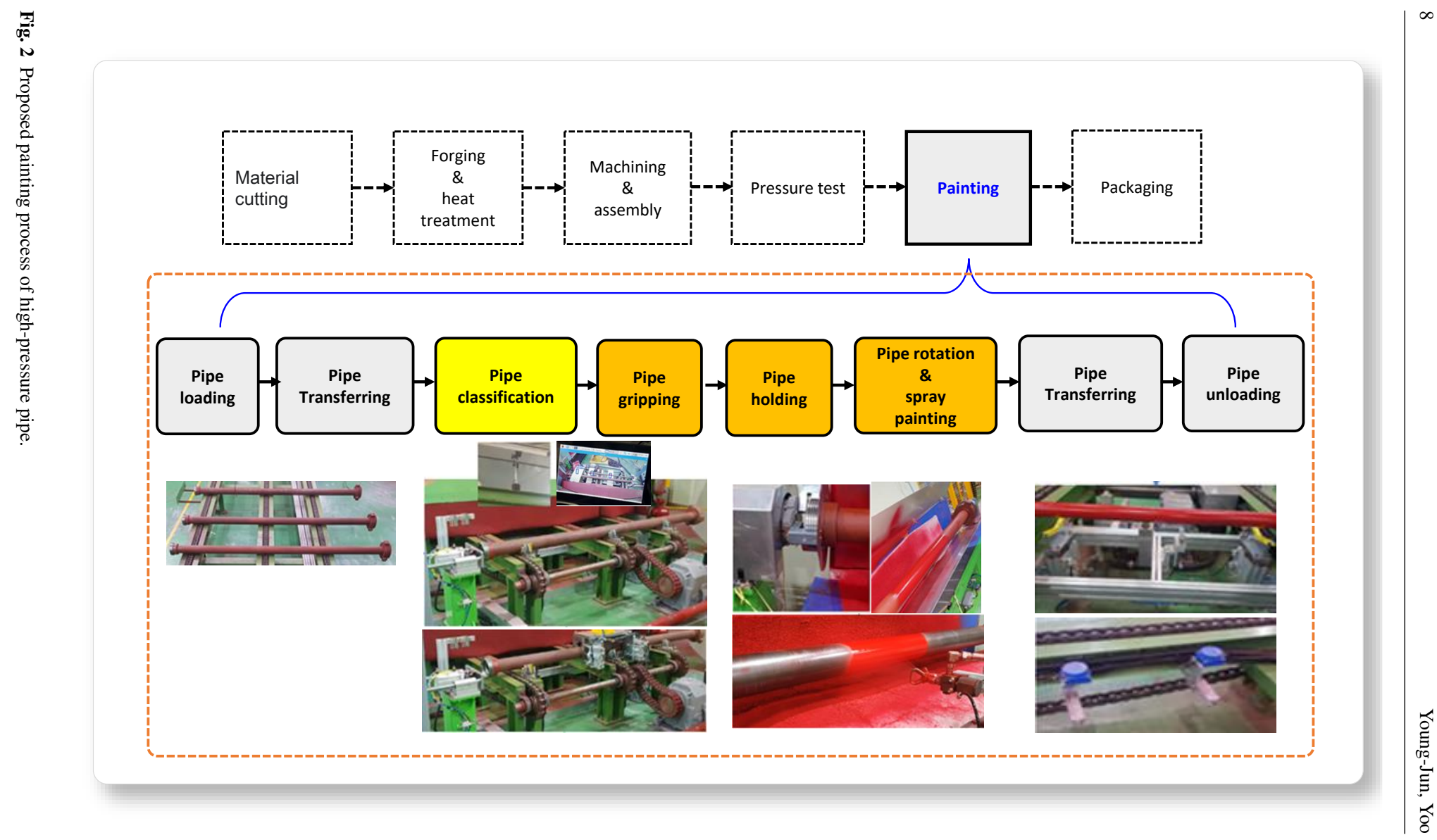




\section{Proposed methodology}

This study proposes a system and process that recognizes the thickness and length using a cost-competitive camera and IoT module to solve the previous section's problem. The process highlighted in yellow and orange in figure 2 is an improvement on the conventional process. In addition, the process was improved for flexible manufacturing of the pipe holding and manual painting parts in figure 1 To this end, the pipe classification process (yellow box in figure 2) and the classification process results are utilized for robot gripping and automatic painting of pipes (orange box in figure

2). Especially, this paper will focus on a system and process (yellow box in figure 2) for pipe classification using an IoT module and USB camera.

3.1 Proposed system hardware and its interfaces

As can be seen in figure 3 the system consists of an IoT module (Raspberry Pi module), an image acquisition unit (camera), and an edge TPU that infers object detection. In addition, the corresponding IoT module communicates with the PLC via Modbus TCP for controlling the robot and equipment so that the recognition result can be interfaced.

The IoT module includes a USB interface that can communicate with the Raspberry Pi camera, an HDMI port that can display images, and an Ethernet port that can communicate the pipe recognition result to other devices. Moreover, it includes a CPU and RAM for calculating the S.W algorithm and also includes wireless com- 


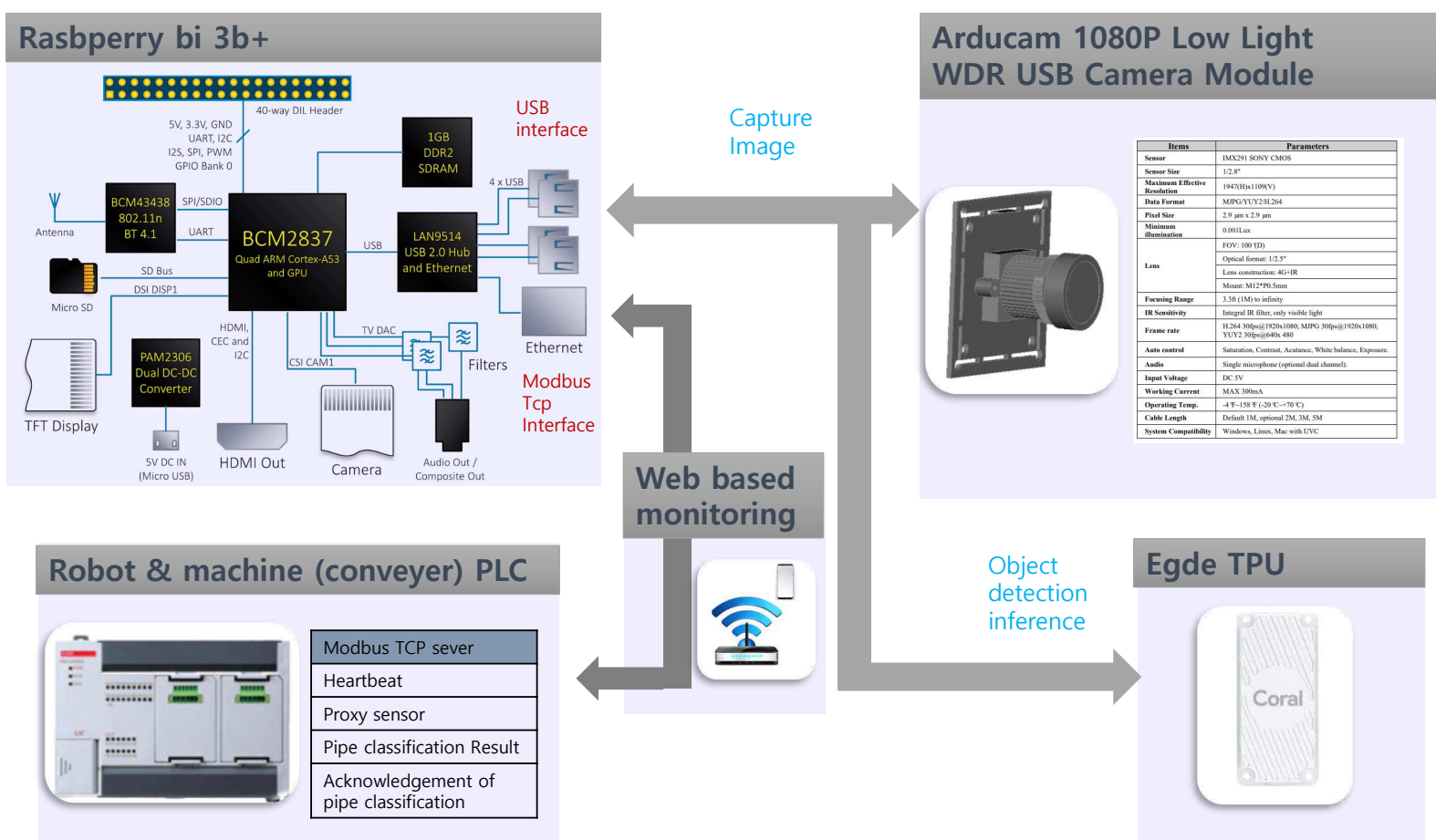


Table 2 USB camera information

\begin{tabular}{ll} 
Specification item & Details \\
\hline \hline Sensor & 1/2.8” IMX291 \\
\hline Resolution & 2MP 1945H x 1109V \\
\hline Data Format & MJPG/YUY2/H.264 \\
\hline Frame Rate & H.264 30fps@ 1920 x 1080 \\
\hline MJPG 30fps@ $1920 \times 1080$ \\
\hline Field of View (FOV) & D $=100^{\circ}$ \\
\hline Focusing Range & $3.3 \mathrm{ft} .(1 \mathrm{M})$ to infinity \\
\hline Power Supply & DC5V \\
\hline Working Current & MAX 300mA \\
\hline Operating Temp & $4^{\circ} \mathrm{F} \sim 158^{\circ} \mathrm{F}\left(-20^{\circ} \mathrm{C} \sim+70^{\circ} \mathrm{C}\right)$. \\
\hline Dimension & $38 \times 38 \mathrm{~mm}(\mathrm{Hole} \mathrm{pitch} 28 \times 28 \mathrm{~mm}, 34 \times 34 \mathrm{~mm})$ \\
\hline
\end{tabular}

Table 3 IoT board information.

\begin{tabular}{ll} 
Specification item & Details \\
\hline \hline System on Chip & Broadcom BCM2837 \\
\hline CPU & 4x ARM Cortex-A53 1.2GHz \\
\hline GPU & Broadcom VideoCore IV \\
\hline RAM & 1GB LPDDR2 (900MHz) \\
\hline Networking & 10/100 Ethernet, 2.4GHz 802.11n wireless \\
\hline Storage & microSD \\
\hline GPIO & 40 -pin header, populated
\end{tabular}


munication. The camera module transmits images to the IoT module.

The specifications of the USB camera for acquiring the pipe image are specified in Table 2, the IoT module connected to the USB camera. The specifications of the IoT module are specified in Table 3. The initial image obtained from the camera is transmitted in $1920 \times 1080$ resolution through proposed hardware interfaces to classify the pipe. Then, the IoT module and the camera are interfaced with USB, and the pipe classification is judged at least once every 5 seconds.

Object detection is used in the proposed system to cross-check whether the pipe is positioned for robot gripping by the pipe image. However, object detection needs a lot of computation resources to infer results, so to speed up deep learning inference, the edge TPU module is interfaced with the IoT module.

The result values obtained by the proposed system are linked to the robot and equipment PLC, and the result values can be checked on the web. As a Modbus TCP server in the equipment PLC, four addresses were used for Modbus TCP communication. The first address is a heartbeat address that flickers every second to check if communication is alive. The second address is an address that shows whether a signal from the proximity sensor gives signals whether the pipe has arrived at the gripper's location. When the signal is "ON" at this address, the pipe classification algorithm is executed. The third address is the address that informs the pipe result recognized by the IoT module. Finally, the last address is an address indicating that classification is complete. When this address is changed to "ON," the robot flexibly changes the gripper position according to the type of pipe and picks the pipe. 
3.2 Proposed pipe classification process

As shown in figure 2 this paper proposes a process for classifying pipe types. First, the image is acquired through the USB camera, processed by the Raspberry Pi, and the pipe from the proximity sensor is reconfirmed through the object detection algorithm. Next, the proposed process detects a line in three regions of interest (ROI) and then determines the length and diameter of the pipe based on the result values of the line. Finally, the classification result is interfaced with the PLC of the robot gripping the pipe and the painting automation equipment to paint for the type of pipe flexibly.

\subsubsection{Pipe classification algorithm}

Figure 4 shows the pipe classification process. After the pipe is transferred from the conveyor belt, the proximity sensor is activated when the robot reaches the position to grip. When the proximity sensor is turned on, the camera image is captured. If it does not work, the conveyor continues to operate. After that, the object detection algorithm runs to cross-check whether the pipe has reached the gripper area.

When it is confirmed that the pipe is transferred from the conveyor in the proximity sensor and the object detection algorithm, the line detection algorithm proceeds in the ROI area of 3 parts. For example, a line is detected in Area 1, and it determines a $10 \mathrm{ft}$. pipe. If it is not detected, check the line detected in Area 2. If a straight line is detected in Area 2, it is an $8 \mathrm{ft}$. pipe. If it is not detected, a line is detected in Area 3. When a line is detected in Area 3, the pipe is classified as a $6 \mathrm{ft}$. pipe.

The diameter of the pipe is determined through the straight lines detected in Area 3. The diameter is calculated through the average of the y-coordinates of the detected 


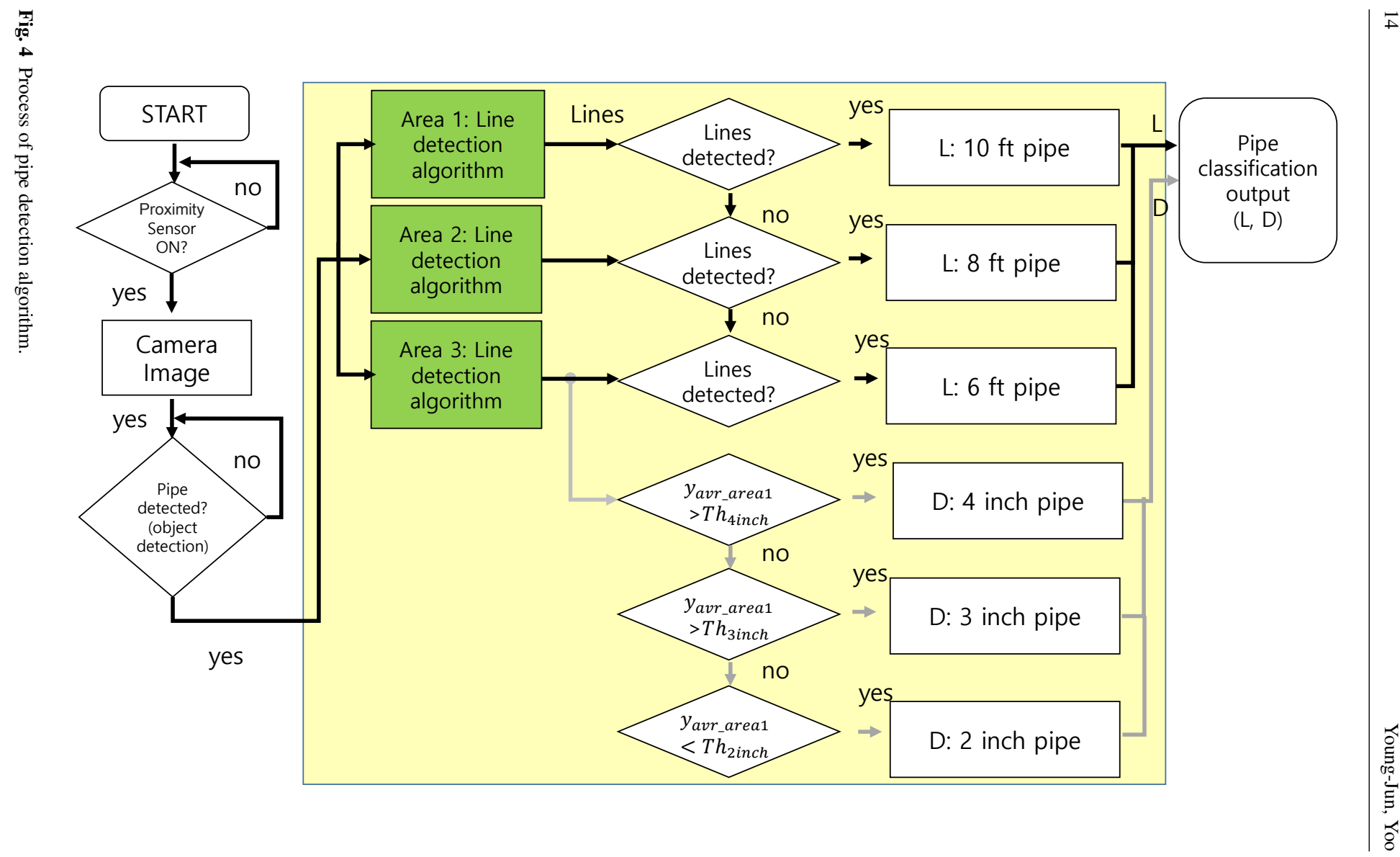




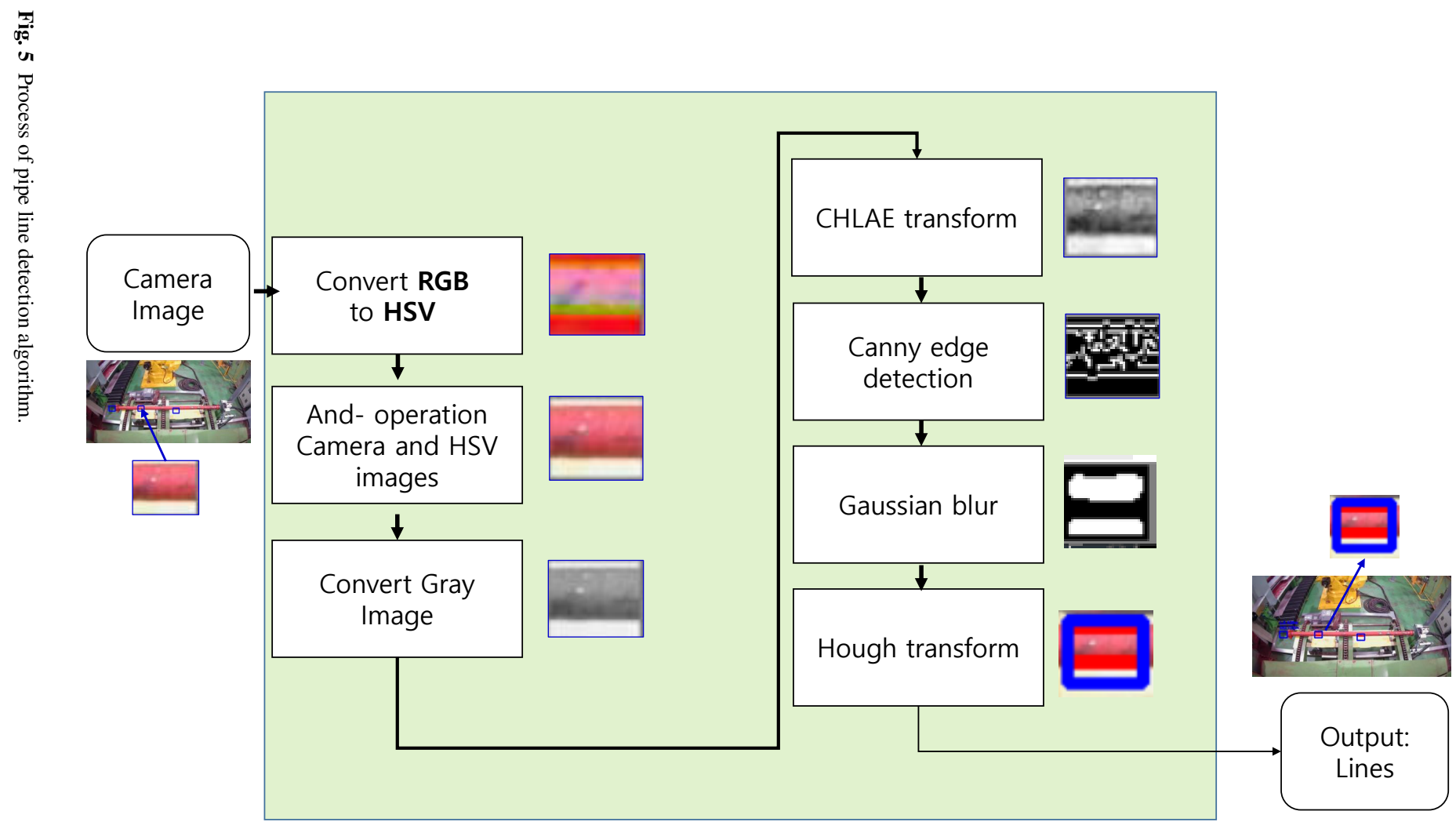


straight lines. According to the diameter of each pipe, the threshold value of 4 inches is defined as $T h_{4 i n c h}, 3$ inches is $T h_{3 i n c h}$, and 2 inches is $T h_{2 i n c h}$, respectively. For example, if the average of the $y$-coordinate is greater than $T h_{4} i n c h$, the 4 inches pipe is classified. Also, if the average of $y$-coordinates is smaller than $T h_{4 i n c h}$ and larger than $T h_{3 i n c h}$, it is classified as a 3-inch pipe. Moreover, if the image pixel of the $y$ coordinate average is smaller than $T h_{2 i n c h}$, it is classified as a 2-inch pipe.

When the results of each pipe's diameter (D) and length (L) come out, the classification results are sent to the PLC of the equipment for pipe gripping and automated painting in figure 2 Through this, the painting process can be applied flexibly even if the type of pipe changes on the conveyor.

\subsubsection{Object detection algorithm}

The model used in the object detection algorithm is the Yolov3-tiny model, and the $416 \times 416$ image is input. In this paper, the number of classes is one, and the name of the class is "PIPE." The proposed system cross-checks whether the pipe has arrived at the grip position through the object detection model, thereby securing the reliability of the pipe recognition algorithm.

\subsubsection{Line detection algorithm}

This subsection describes the line detection algorithm for classifying pipes (highlighted by the green box in Fig. 4). The algorithm for extracting lines from the ROI of the camera image consists of seven steps. 
RGB to HSV conversion is performed to extract the corresponding color of the pipe from the RGB image. Since the pipe color is specified as red, only the color is extracted after HSV conversion and operation with the existing image. After the red color is extracted, it is converted into a gray image. Contrast-limited adaptive histogram equalization (CLAHE) [12] was applied in this study to increase the sharpness of gray images and to be robust against illumination under the pie surface condition. Next, apply Canny edge detection is applied to the image for detecting lines. After that, Gaussian blur is applied to remove the noise. Finally, a straight line is detected through the Hough transform. The detected straight line is used to determine the pipe class in three ROIs, as shown in figure 4

\subsubsection{PLC interface for flexable manufacturing system}

The sequence program through image-based pipe recognition is interfaced with the painting equipment PLC, enabling the robot to install depending on the type of pipe. The development module and PLC interface are configured as shown in figure 3 , and communication between the development module and the equipment PLC is configured through Modbus TCP communication. Four addresses are used for communication, the heartbeat indicating that communication is alive (ON/OFF every 1 second), the point at which the pipe comes from the conveyor belt to the place where the robot installs and starts to recognize the pipe type. When the pipe IN signal (transmitted from PLC to the development module) and pipe in signal comes in, the algorithm developed in the previous chapter determines the pipe type 3 times or more and con- 
sists of a pipe type and a signal indicating that recognition is complete. In addition, the result recognized by the development module is broadcast on the Web, and when a web page is accessed to a specific address, the recognition result and communication address value can be viewed.

\section{Method validation setup}

4.1 Image acquisition Hardware

As shown in Fig. 6, the proposed pipe classification system and process were installed and applied to the painting process. The yellow box shown in figure 6a is a system composed of an IoT module, an edge TPU, and a screen that can monitor it. The yellow box marked in figure $6 \mathrm{~b}$ shows a USB camera to acquire an image and a jig to fix it. Finally, figure $6 \mathrm{c}$ is a router for Modbus TCP communication between the IoT module and PLC, and the result can be checked through the router through the web.

\section{2 object detection training results}

A deep learning model for pipe recognition was established from the images acquired from the proposed IoT module and USB camera, and pipe location labeling was performed based on the acquired image. The Yolov3-tiny model was trained 2000 times, and the loss values were 0.5260 and $98.3 \%$ mAP. The object detection result makes it possible to recheck whether the pipe is transferred from the conveyor belt to the position where the robot will grip. 


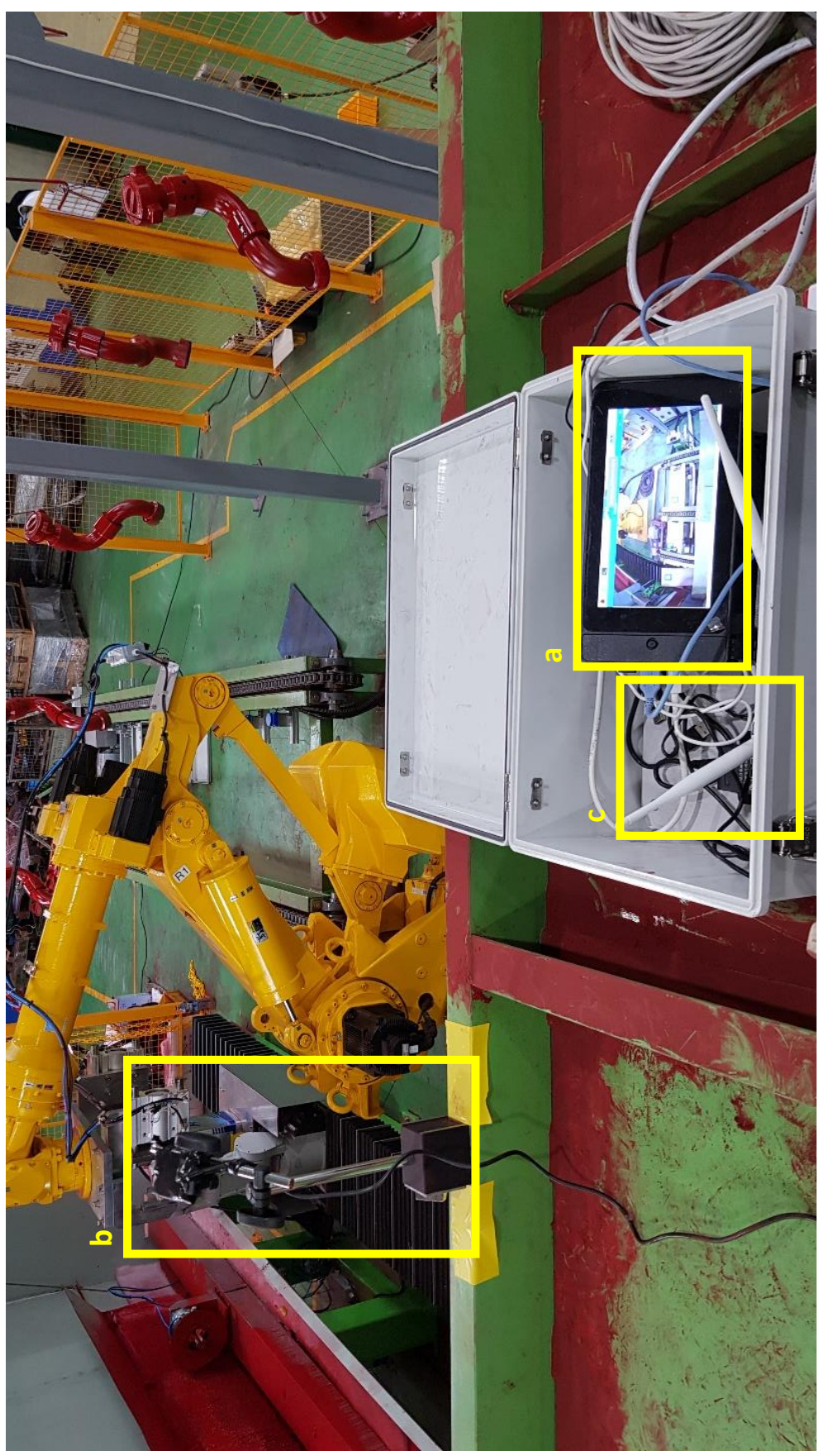

Fig. 6 Field application of the proposed flexible manufacturing system: (a) IoT module, (b) USB camera, and (c) router for communication. 


\section{Results and discussion}

5.1 Deep learning-based object detection results

Figure 8 shows the result of object detection of the pipe. In figure $8 \mathrm{abc}$, the part marked with a purple box is the area recognized as a pipe. Pipe classification proceeds if the proximity sensor of the equipment that is held is recognized as $\mathrm{ON}$ when the area where the pipe is recognized is to hold the pipe to the robot gripper. In figure $8 \mathrm{abc}$, it can be seen that all pipes are detected.

\subsection{Pipe classification results}

Figure 9 shows the results of applying the process to a 3 inches, $10 \mathrm{ft}$. pipe, and 4 inches $10 \mathrm{ft}$. pipe. A blue box indicates the ROI of the proposed system. The pipe type is determined after performing line detection in each area from the left to Area 1, Area 2, and Area 3. The first and second blue box from the left is a module that determines the length of the pipe. When a pipe exists, lines are detected. If the lines are detected in the first and second boxes, the proposed system results in a $10 \mathrm{ft}$. pipe. If the first straight line is not detected, and the second is detected, the system determines an $8 \mathrm{ft}$. pipe. Finally, if only the lines are detected in the 3rd box, it is recognized as $6 \mathrm{ft}$. pipe. The diameter is determined through the y-pixels of Area 3, and in the case of 2 inches, 3 inches, and 4 inches, the average position of the straight line of the corresponding pixel is the 6th, 7th, and 8th pixels, respectively.

In the case of 3 inches and $10 \mathrm{ft}$. (figure $9 \mathrm{a}$ ) pipe, lines were detected in all areas and determined to be $10 \mathrm{ft}$. $(3.048 \mathrm{~m})$, and the average position of the straight line in 
area 3 was indicated as number 7 and determined as 3 inches $(0.152 \mathrm{~m})$. Also, in figure 9p, all straight lines were detected and classified as the longest pipe, $10 \mathrm{ft}$. (3.048 $\mathrm{m})$, and the average position of the straight line was calculated as nine and classified as a 4 inch $(0.203 \mathrm{~m})$ pipe.

\subsection{Monitoring results}

Figure 10 shows checking the pipe recognition process by accessing the result transmitted to the web from a mobile phone. On the left, after the pipe contacts the proximity sensor, before sending the pipe in signal from PLC, after the pipe in signal is turned on as shown in the middle of figure 9 After recognizing the pipe, as shown on the right side of figure 10 , the third pipe ( 2 inches and 10ft) is recognized, and it is a picture that informs the PLC that the classification process is finished. Based on this web monitoring system, it is possible to monitor the results of pipe classification in real-time, and it can help workers make quick decisions in emergencies such as abnormal situations

\section{Conclusion}

In this paper, an IoT module that recognizes nine types of pipes used in the painting process was developed. Recognizes nine types of pipes, three types of diameter (2 inches, 3 inches, and 4 inches) and three types of lengths (6ft, $8 \mathrm{ft}$, and 10ft) used in the process, and sets the pipe type to the robot An IoT module that informs has been developed. The IoT module that recognizes the type interfaces with the painting 
robot, and the sequence control code for painting by type of pipe is executed on the painting robot, enabling one-coat painting to proceed. Through the automation of this module and the painting robot, nine types of pipes can be painted automatically. In other words, it is possible to paint products of different diameters and lengths through the robot work program flexibly. The advantage of this IoT module is that it is an IoT module with price competitiveness and can be used in processes using similar object size recognition.

\section{Declarations}

Funding This study has been conducted with the support of the Ministry of SMEs and Startups as "Development of 1-coating paint method and process innovation based on the shape recognition of ultra high pressure pipe".

Conflicts of interest The author declare that they have no competing interests Availability of data and material "Not applicable"

Code availability "Not applicable"

Consent to participate "Not applicable"

Consent for publication "Not applicable"

\section{References}

1. Yepeng Fan, Jianzhong Yang, Jihong Chen, Pengcheng Hu, Xiaoyu Wang, Jianchun Xu, Bin Zhou (2021) A digital-twin visualized architecture for Flexible Manufacturing System, Journal of Manufacturing Systems, 60: 176-201. 
2. Jenny L. Diaz C., Carlos Ocampo-Martinez (2021) Non-centralised control strategies for energyefficient and flexible manufacturing systems, Journal of Manufacturing Systems, 59: 386-397.

3. JoseDaniel Hernandez, Edgar Schneider Cespedes, David Andres Gutierrez, David Sanchez-Londoño, Giacomo Barbieri, Sepideh Abolghasem, David Romero, Luca Fumagalli (2020) Human-ComputerMachine Interaction for the Supervision of Flexible Manufacturing Systems: A Case Study, IFACPapersOnLine, 53(2): 10550-10555.

4. Wei Liu, Dragomir Anguelov, Dumitru Erhan, Christian Szegedy, Scott Reed, Cheng-Yang Fu, Alexander C. Berg (2015) SSD: Single Shot MultiBox Detector, arXiv:1512.02325v5.

5. Andrew G. Howard, Menglong Zhu, Bo Chen, Dmitry Kalenichenko, Weijun Wang, Tobias Weyand Marco, Andreetto Hartwig Adam (2017) MobileNets: Efficient Convolutional Neural Networks for Mobile Vision Applications, arXiv:1704.04861v1.

6. Shaoqing Ren, Kaiming He, Ross Girshick, Jian Sun (2015) Faster R-CNN: Towards Real-Time Object Detection with Region Proposal Networks, arXiv:1506.01497

7. Joseph Redmon, Santosh Divvala, Ross Girshick, Ali Farhadi, You Only Look Once: Unified, RealTime Object Detection, arXiv:1506.02640, (2015).

8. Zhong-Qiu Zhao, Peng Zheng, Shou-Tao Xu, Xindong Wu (2019) Object Detection with Deep Learning: A Review, IEEE Transactions on Neural Networks and Learning Systems 30(11): 3212-3232.

9. Farzeen Munir, Shoaib Azam, Muhammd Aasim Rafique, Ahmad Muqeem Sheri (2021) Moongu Jeon, Witold Pedrycz, Exploring Thermal Images for Object Detection in Underexposure Regions for $\mathrm{Au}-$ tonomous Driving, arXiv:2006.00821v2.

10. Hao Zhang, Xianggong Hong (2019) Recent progresses on object detection: a brief review, Multimedia Tools and Applications 78: 27809-27847.

11. Ju Sik Kim, Kyu Nam Choi, and Sung Woo Kang (2021) Infrared Thermal Image-Based Sustainable Fault Detection for Electrical Facilities, Sustainability, 13(2): 557.

12. Ju Sik Kim, Kyu Nam Choi, and Sung Woo Kang, Improved Method for Enhancing Dark Images based on CLAHE and Morphological Reconstruction, Procedia Computer Science,165, (2019), 391398. 


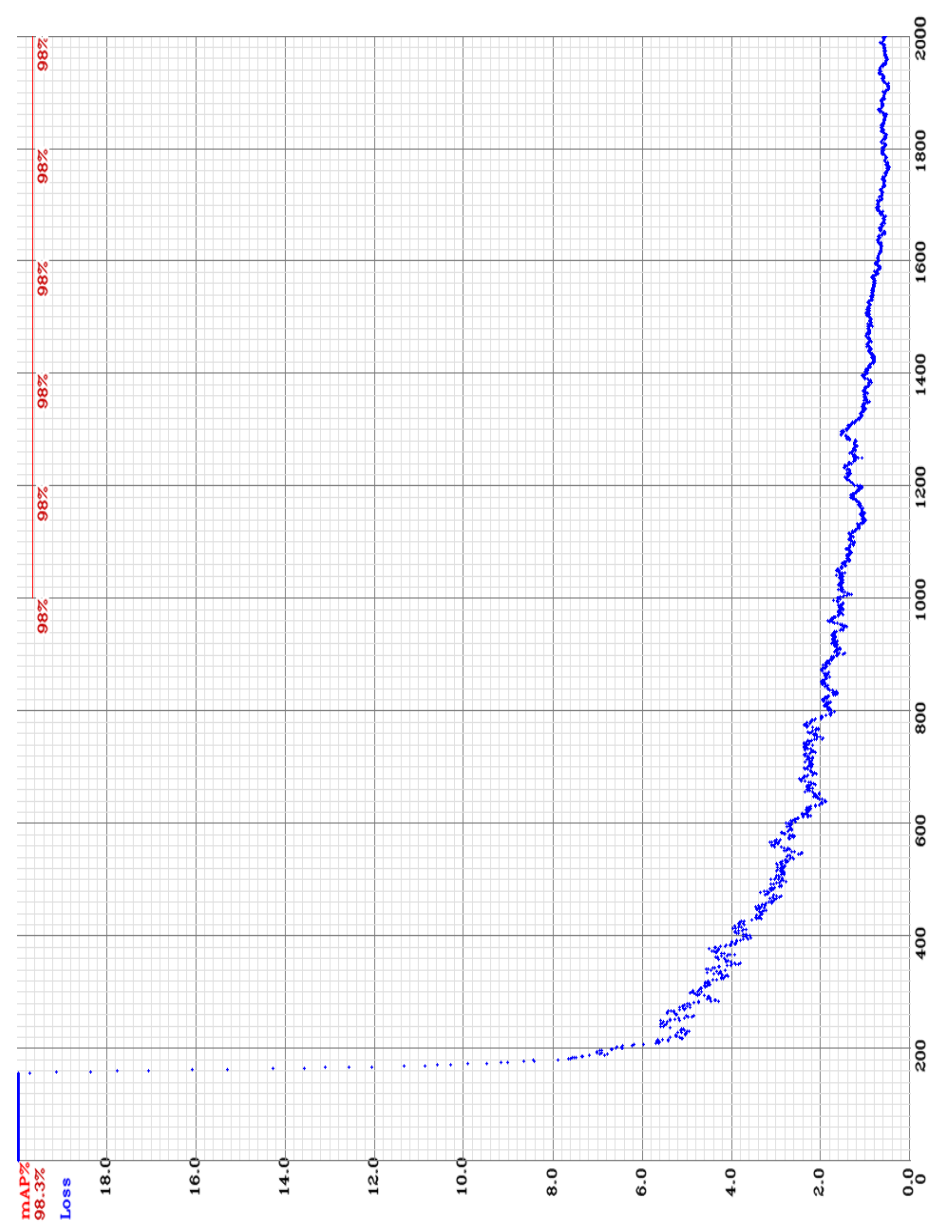

Fig. 7 Object detection training results. 

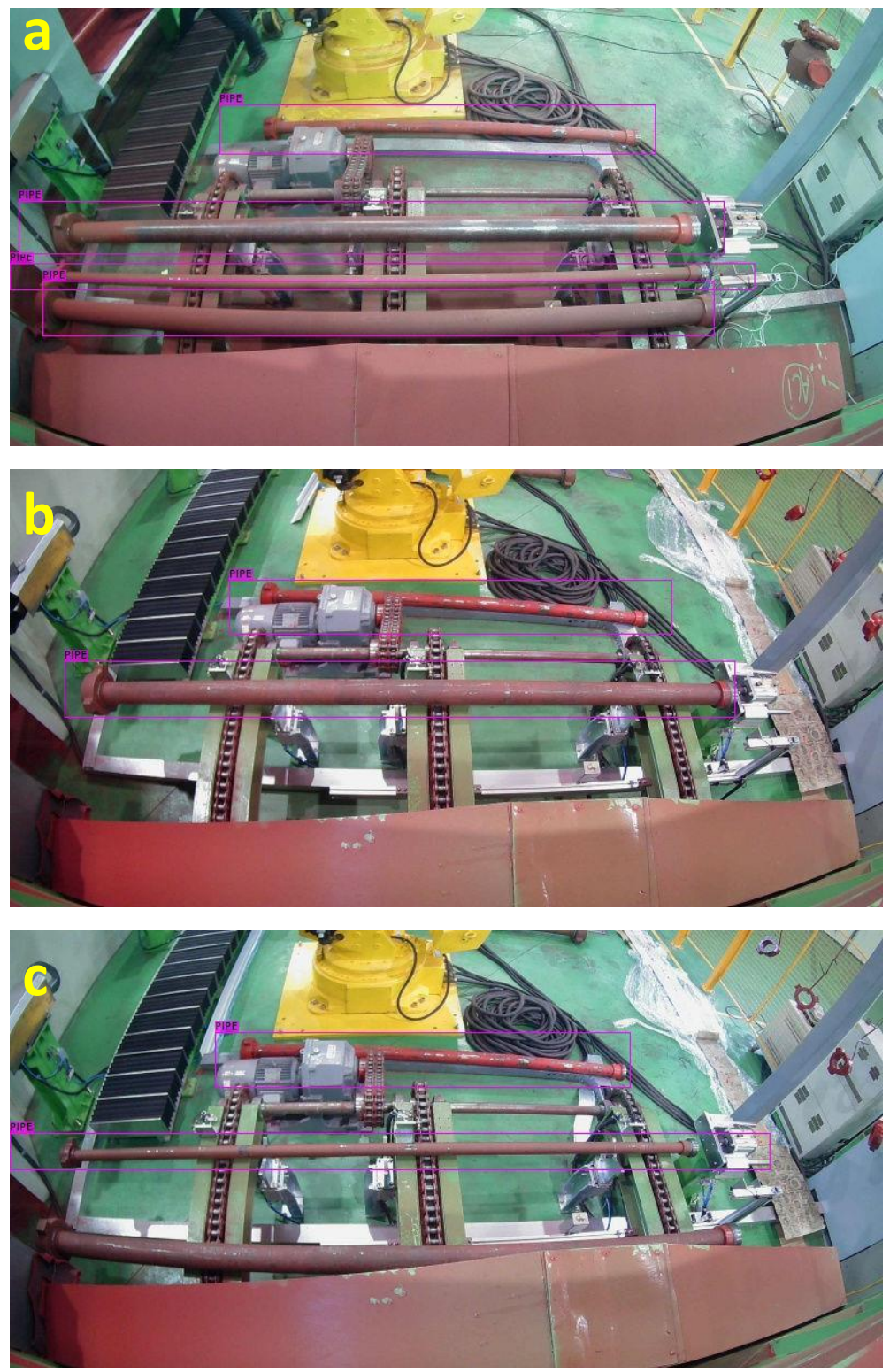

Fig. 8 Object detection results at the holding module for gripping: (a) 4 inches, $10 \mathrm{ft}$. pipe, (b) 3 inches, $10 \mathrm{ft}$. pipe, and (c) 2 inches, $10 \mathrm{ft}$. pipe. 

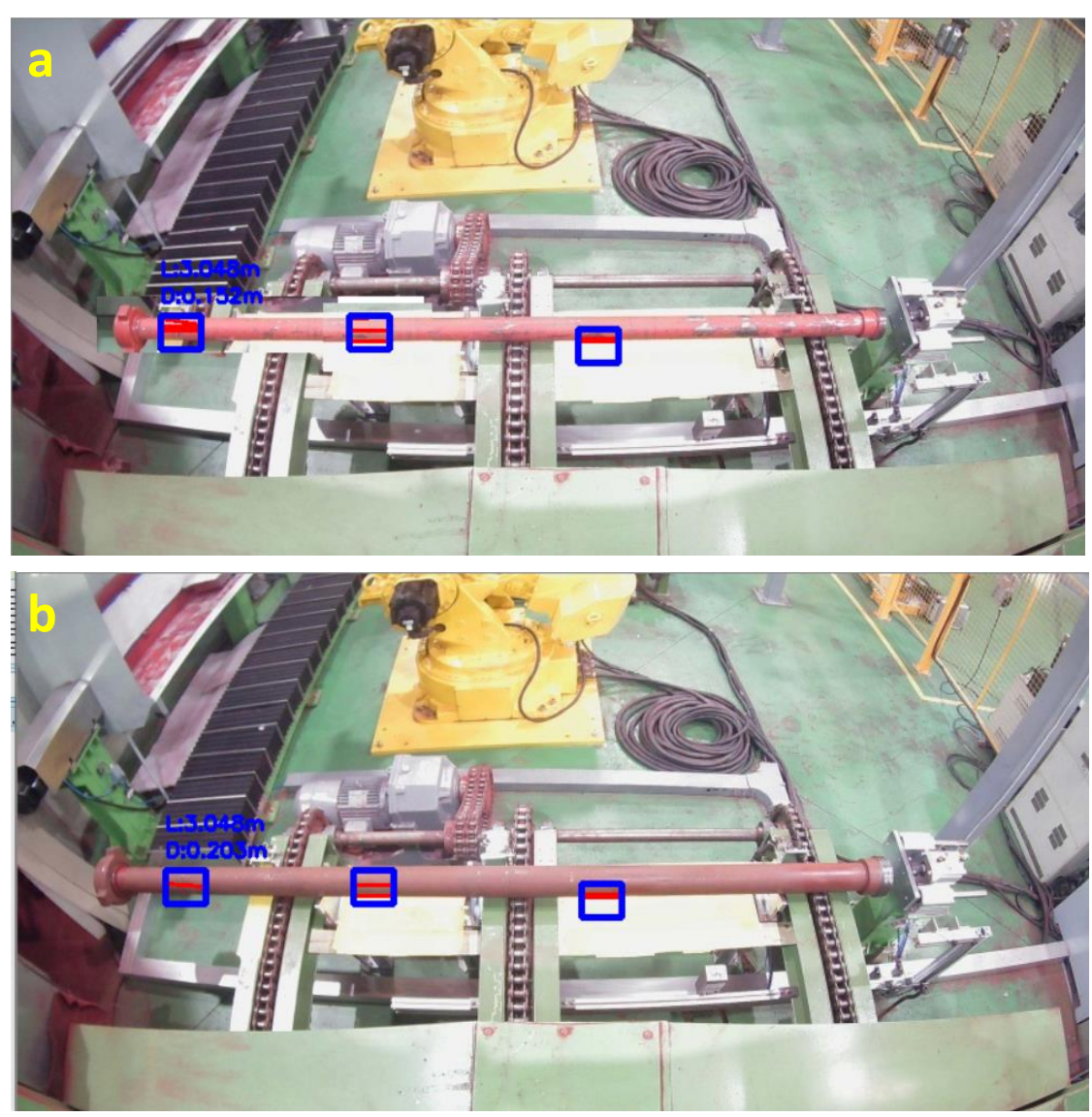

Fig. 9 Pipe classification examples: (a) 3 inches, $10 \mathrm{ft}$. pipe and (b) 4 inches, $10 \mathrm{ft}$. pipe. 


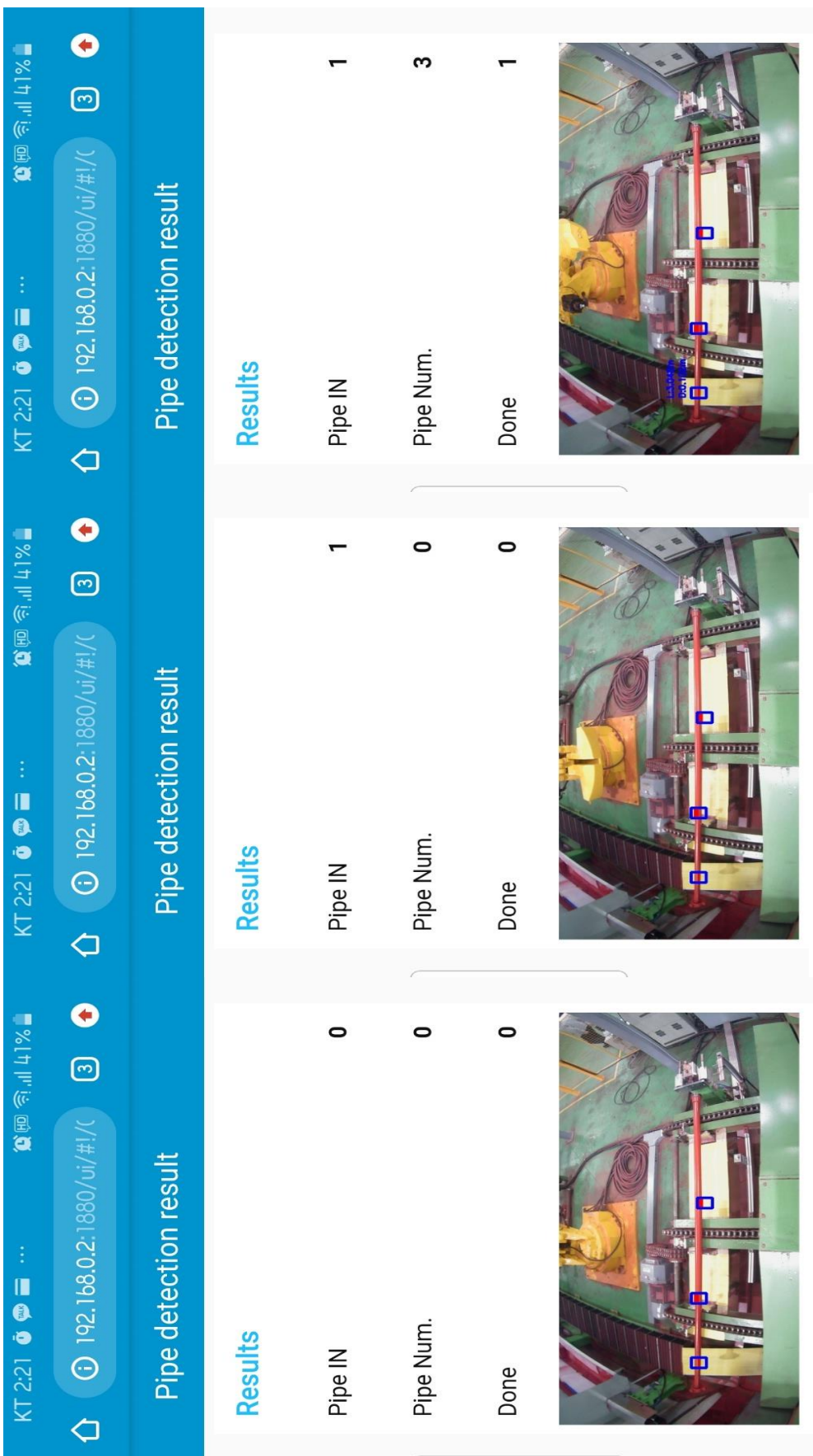

Fig. 10 web monitoring results. 


\section{Supplementary Files}

This is a list of supplementary files associated with this preprint. Click to download.

- paintingFMS.avi 